\title{
Prevalence of type III secretion genes in clinical and environmental isolates of Pseudomonas aeruginosa
}

\author{
Heather Feltman, ${ }^{1}$ Grant Schulert, ${ }^{1}$ Salman Khan, ${ }^{1}$ Manu Jain, ${ }^{2}$ \\ Lance Peterson ${ }^{3}$ and Alan R. Hauser ${ }^{1,2}$
}

Departments of

Microbiology/

Immunology ${ }^{1}$, Medicine ${ }^{2}$ and Pathology ${ }^{3}$,

Northwestern University,

Chicago, IL 60611, USA
Author for correspondence: Alan R. Hauser. Tel: +1 312503 1044. Fax: +1 3125031339. e-mail: ahauser@northwestern.edu

The type III secretion system of Pseudomonas aeruginosa transports four known effector proteins: ExOS, ExOT, ExoU and ExoY. However, the prevalence of the type III secretion system genes or the effector-encoding genes in clinical and environmental isolates of $P$. aeruginosa has not been well studied. Southern hybridization analyses and PCR were performed on over 100 $P$. aeruginosa isolates to determine the distribution of these genes. Clinical isolates were obtained from urine, endotracheal, blood and wound specimens, from the sputum of cystic fibrosis (CF) patients, and from non-hospital environmental sites. The popB gene was used as a marker for the presence of the large chromosomal locus encoding the type III secretion machinery proteins. Each isolate contained the popB gene, indicating that at least a portion of this large chromosomal locus was present in all isolates. Likewise, each isolate contained exoT-like sequences. In contrast, the exoS, exoU and exo $Y$ genes were variable traits. Overall, $72 \%$ of examined isolates contained the exoS gene, $28 \%$ contained the exo $U$ gene, and $89 \%$ contained the exo $Y$ gene. Interestingly, an inverse correlation was noted between the presence of the exoS and exoU genes in that all isolates except two contained either exoS or exoU but not both. No significant difference in exoS, exoU or exoY prevalence was observed between clinical and environmental isolates or between isolates cultured from different disease sites except for CF respiratory isolates. CF isolates harboured the exoU gene less frequently and the exoS gene more frequently than did isolates from some of the other sites of infection, including the respiratory tract of patients without CF. These results suggest that the $P$. aeruginosa type III secretion system is present in nearly all clinical and environmental isolates but that individual isolates and populations of isolates from distinct disease sites differ in their effector genotypes. The ubiquity of type III secretion genes in clinical isolates is consistent with an important role for this system in human disease.

Keywords: ExoU, ExoY, ExoS, ExoT, cystic fibrosis

\section{INTRODUCTION}

Pseudomonas aeruginosa is a Gram-negative bacterium that causes a variety of diseases in compromised hosts. For example, this organism first colonizes the lungs of children with cystic fibrosis (CF) between 5 and 9 years of age (Pedersen et al., 1986) and can subsequently be

Abbreviation: $\mathrm{CF}$, cystic fibrosis. cultured from the sputum of approximately $80 \%$ of adults over the age of 25 (Fitzsimmons, 1993). Once established within the lungs, $P$. aeruginosa usually causes episodic bouts of pneumonia that lead to progressive irreversible lung injury and ultimately death. Another group of individuals particularly prone to infections by this organism is hospitalized patients. In the United States, it is estimated that P. aeruginosa is responsible for $17 \%$ of nosocomial pneumonias, $11 \%$ of nosocomial urinary tract infections, $8 \%$ of surgical 
wound infections and $3 \%$ of central-line-associated bloodstream infections (National Nosocomial Infections Surveillance System, 1996). Given the frequency of $P$. aeruginosa infections and the increasing resistance of this organism to conventional antibiotics (Fluit et al., 2000; Hanberger et al., 1999), it is not surprising that $P$. aeruginosa illnesses are associated with significant morbidity and mortality. For example, $P$. aeruginosa nosocomial pneumonia is associated with crude mortality rates as high as $70 \%$ overall (Pennington, 1995) and as high as $90 \%$ in mechanically ventilated patients (Fagon et al., 1989); attributable mortality rates are approximately $40 \%$ (Fagon et al., 1993).

To cause such severe disease, $P$. aeruginosa utilizes a large number of secreted and cell-associated virulence factors. These include exotoxin A, phospholipase, alkaline protease, elastase, pyocyanin, pili, flagella and lipopolysaccharide (Salyers \& Whitt, 1994). Many of these factors are variable traits - they are produced by some clinical isolates but not by others. An important and recently recognized virulence determinant of $P$. aeruginosa is the type III secretion system (Kang et al., 1997; Yahr et al., 1996b). The genes encoding the secretion, translocation and regulatory machinery of this system are clustered together in the $55 \mathrm{~min}$ region of the $P$. aeruginos a chromosome and have been designated psc, pcr, exs and pop genes (reviewed by Frank, 1997). The $p s c$ and $p c r$ genes primarily encode components of the bacterial secretion apparatus whereas the exs genes and their products are involved in regulation of this complex system. The two pop genes encode proteins that are essential for the translocation of effector proteins into host cells. The $p o p B$ gene encodes PopB (also known as PepB) and the $p o p D$ gene encodes PopD (also known as PepD).

In contrast to the clustered genes encoding the type III transport machinery, the genes encoding the type III effector proteins appear to be scattered throughout the chromosome (Stover et al., 2000; the Pseudomonas Genome Project, http://www.pseudomonas.com). To date, four effector proteins have been identified: ExoS, ExoT, ExoU (also called PepA) and ExoY. ExoS possesses two distinct functional domains. The aminoterminus contains a GTPase-activating domain for Rho GTPases and results in disruption of actin microfilaments and subsequent cell rounding (Frithz-Lindsten et al., 1997; Goehring et al., 1999; Pederson et al., 1999; Vallis et al., 1999). The carboxyl-terminus has ADPribosyltransferase activity directed towards Ras and other proteins and is cytotoxic to eukaryotic cells (Frithz-Lindsten et al., 1997; Henriksson et al., 2000; Olson et al., 1997, 1999; Vincent et al., 1999). In addition, ExoS may play a role in modulating bacterial internalization by eukaryotic cells (Cowell et al., 2000; Fleiszig et al., 1997; Frithz-Lindsten et al., 1997). ExoT is also an ADP-ribosyltransferase but has only $0 \cdot 2 \%$ of the catalytic activity of ExoS (Yahr et al., 1996a). Like ExoS, it is a GTPase-activating protein for Rho GTPases and inhibits bacterial internalization by eukaryotic cells
(Cowell et al., 2000; Garrity-Ryan et al., 2000; Krall et al., 2000). Although the mechanism of action of the third effector protein, ExoU, is unclear, laboratory studies suggest that this factor is quite important in disease. ExoU mediates killing of a variety of mammalian cell types in vitro, including macrophages, epithelial cells and fibroblasts (Coburn \& Frank, 1999; FinckBarbancon et al., 1997; Fleiszig et al., 1997; Hauser \& Engel, 1999; Hauser et al., 1998; Vallis et al., 1999). Furthermore, isogenic mutants that do not produce or secrete ExoU are defective in virulence in a mouse model of pneumonia (Finck-Barbancon et al., 1997; Hauser et al., 1998). Unlike the wild-type strain, an isogenic mutant defective in secretion of ExoU and ExoT did not cause sepsis in a rabbit model of pneumonia (Kurahashi et al., 1999). The fourth effector, ExoY, is an adenylate cyclase that elevates the intracellular cAMP levels in eukaryotic cells and causes rounding of certain cell types (Vallis et al., 1999; Yahr et al., 1998).

Interestingly, the genes encoding some $P$. aeruginosa type III effector proteins are variable traits (i.e. they are found in some isolates but not in others) (FinckBarbancon et al., 1997; Fleiszig et al., 1997; Hauser et al., 1998). The distribution of these genes amongst clinical isolates of $P$. aeruginosa remains to be elucidated. Furthermore, the prevalence of effector genes in populations of isolates from different disease sites has not been systematically and thoroughly explored. In this report, we investigate the prevalence of type III effector genes in populations of $P$. aeruginosa isolates obtained from the blood, wounds, respiratory tracts and urinary tracts of patients as well as from non-hospital environmental sources.

\section{METHODS}

Bacterial strains and growth conditions. An initial collection of 95 P. aeruginosa clinical isolates was obtained from patients at Northwestern Memorial Hospital in Chicago between March 1999 and December 2000. Consecutive isolates were collected and analysed using a protocol approved by the Northwestern University Institutional Review Board. Isolates were identified as $P$. aeruginosa by the Clinical Microbiology Laboratory at Northwestern Memorial Hospital using standard protocols (Gilardi, 1991). To prevent overrepresentation of certain strains, only one isolate per patient was included in the analysis. A second collection of 20 consecutive CF respiratory isolates and 20 consecutive non-CF respiratory isolates was analysed to further examine the distribution of exoU and exoS. These isolates had been previously collected from patients at Moffitt-Long Hospital at the University of California, San Francisco, between November 1997 and October 1998 and stored as frozen stocks. The origins of the $P$. aeruginosa environmental isolates are described in Table 1. Isolates were minimally passaged on Vogel-Bonner minimal media plates (Vogel \& Bonner, 1956) or Luria-Bertani (LB) agar plates and were stored at $-70{ }^{\circ} \mathrm{C}$ in $25 \%$ glycerol.

P. aeruginosa strain PA103, which contains exoU, exoT and exoY genes (Finck-Barbancon et al., 1997; Hauser et al., 1998; data not shown), and strain 388, which contains exoS, exoT and exoY genes (Yahr et al., 1996a, 1998), were used as controls in Southern hybridization experiments. 
Table 1. Environmental isolates used in this study

\begin{tabular}{|c|c|c|c|}
\hline Isolate & $\begin{array}{c}\text { Original } \\
\text { designation }\end{array}$ & Origin & Source \\
\hline EV1 & Env 1 & Green onion & $\begin{array}{l}\text { David P. Speert, University of } \\
\text { British Columbia }\end{array}$ \\
\hline EV2 & Env 11 & Potato & David P. Speert \\
\hline EV3 & Env 12 & Green pepper & David P. Speert \\
\hline EV4 & Env 14 & Green pepper & David P. Speert \\
\hline EV5 & Env 16 & Tomato & David P. Speert \\
\hline EV6 & Env 32 & Zucchini & David P. Speert \\
\hline EV7 & Env 37 & River isolate & David P. Speert \\
\hline EV8 & Env 47 & River isolate & David P. Speert \\
\hline EV9 & Env 58 & River isolate & David P. Speert \\
\hline EV10 & Env 62 & River isolate & David P. Speert \\
\hline EV11 & 15524 & Soil & ATCC \\
\hline EV12 & 21472 & Soil from oilfield & ATCC \\
\hline EV13 & 15523 & Soil & ATCC \\
\hline EV14 & 7700 & Well water & ATCC \\
\hline EV15 & 7701 & Well water & ATCC \\
\hline EV16 & 14886 & Soil & ATCC \\
\hline EV17 & 21776 & Soil & ATCC \\
\hline EV18 & LPL5 & Lake & $\begin{array}{l}\text { Robert V. Miller, Oklahoma } \\
\text { State University }\end{array}$ \\
\hline EV19 & LLPA10 & Lake & Robert V. Miller \\
\hline EV20 & JB2 & $\begin{array}{l}\text { Polychlorinated biphenyl- } \\
\text { contaminated soil }\end{array}$ & $\begin{array}{l}\text { William J. Hickey, University } \\
\text { of Wisconsin }\end{array}$ \\
\hline
\end{tabular}

Table 2. Primers used for detection of type III secretion genes

\begin{tabular}{|c|c|c|}
\hline & $\begin{array}{c}\text { Primers for amplification of probes for Southern } \\
\text { hybridization assays* }\end{array}$ & Primers for PCR-based detection assays \\
\hline \multirow[t]{2}{*}{ exoU } & $5^{\prime}-$ AGCGTTAGTGACGTGCG-3' & 5'-AGCGTTAGTGACGTGCG-3' \\
\hline & 5'-GCGCTCGCTCTTCAACGG-3' & 5'-GCGCATGGCATCGAGTAACTG-3' \\
\hline \multirow[t]{2}{*}{ exoS十 } & 5'-TTTGGATCCATGCATATTCAATCGCTTCAGCAGA-3' & 5'-TCAGGTACCCGGCATTCACTACGCGG-3' \\
\hline & 5'-CCССTTAAGTCAGGCCAGGTCGAGGCCGCG-3' & 5'-TCACTGCAGGTTCGTGACGTCTTTCTTTTA-3' \\
\hline \multirow[t]{2}{*}{ exoT十 } & 5'-TTTGGATCCATGCATATTCAATCGCTTCAGCAGA-3' & 5'-TCACTGCAGTTCCGCGTGCTCCGACG-3' \\
\hline & 5'-CCCCTTAAGTCAGGCCAGGTCGAGGCCGCG-3' & 5'-TCAGGTACCTGCTGGTACTCGCCGTT-3' \\
\hline \multirow[t]{2}{*}{ exoY } & 5'-TCCAAGCTTATGCGTATCGACGGTCATC-3' & 5'-TCCAAGCTTATGCGTATCGACGGTCATC-3' \\
\hline & 5'-CCCTCTAGATCAGACCTTACGTTGGAAAAAG-3' & 5'-CGTATCGATCCGAGGGGGGTGTATCTGACC-3' \\
\hline \multirow[t]{2}{*}{$p \circ p B$} & 5'-TTTGGATCCATGAATCCGATAACGCTTG-3' & 5'-TTTGGATCCATGAATCCGATAACGCTTG-3' \\
\hline & 5'-TTTGAATTCTCAGATCGCTGCCGGTCG-3' & 5'-TTTGAATTCTCAGATCGCTGCCGGTCG-3' \\
\hline
\end{tabular}

$* 5^{\prime}$-primers are listed first and $3{ }^{\prime}$-primers are listed last.

† Since the exoS and exoT genes are $80 \%$ identical in nucleic acid sequence, the same primers were used with different templates to generate probes for both genes.

Construction of gene-specific labelled probes for Southern hybridization analyses. Digoxigenin-labelled probes for Southern hybridization analyses were generated using PCR with upstream and downstream primers listed in Table 2. PCR was performed in the presence of digoxigenin-11-dUTP (Roche Molecular Biochemicals). In this way, internal probes of the following sizes were generated : exoU gene, $1 \cdot 1 \mathrm{~kb} ;$ exoS gene, $1.4 \mathrm{~kb}$; exoT gene, $1.4 \mathrm{~kb}$; exoY gene, $1.1 \mathrm{~kb}$; popB gene, $1 \cdot 2 \mathrm{~kb}$. Cloned or chromosomal copies of each gene were used as templates. The following protocol was used to amplify the desired DNA products: $94^{\circ} \mathrm{C}$ for $3 \mathrm{~min}$, then 40 cycles of $94^{\circ} \mathrm{C}$ for $30 \mathrm{~s}, 50^{\circ} \mathrm{C}$ for $30 \mathrm{~s}$, and $72{ }^{\circ} \mathrm{C}$ for $2 \mathrm{~min}$, then a final extension at $72{ }^{\circ} \mathrm{C}$ for $5 \mathrm{~min}$. Amplified and labelled DNA fragments were electrophoresed through agarose gels $(0.8 \%$, $\mathrm{w} / \mathrm{v}$ ), and the appropriately sized fragments were extracted and used as probes in Southern hybridization experiments. 
Southern hybridization analysis. Southern hybridizations were performed using the DIG System (Roche Molecular Biochemicals) in accordance with the manufacturer's instructions. Chromosomal DNA from clinical isolates was purified by the method of Chen \& Kuo (1993). DNA was digested with $B a m \mathrm{HI}$, electrophoresed through agarose gels $(0.8 \%, \mathrm{w} / \mathrm{v})$, and transferred to nylon membranes (Hybond$\mathrm{N}$; Amersham). Following hybridization with labelled probes, membranes were washed twice in $2 \times$ SSC (SSC is $0.15 \mathrm{M}$ $\mathrm{NaCl}, 0 \cdot 015 \mathrm{M}$ sodium citrate, $\mathrm{pH} 7 \cdot 0$ ), $0 \cdot 1 \%$ (w/v) SDS for $15 \mathrm{~min}$ at room temperature followed by two washes in $0.5 \times$ SSC, $0 \cdot 1 \%(\mathrm{w} / \mathrm{v})$ SDS for $15 \mathrm{~min}$ at $68{ }^{\circ} \mathrm{C}$. Membranes were then soaked in blocking buffer followed by incubation with anti-DIG-POD Fab fragments (Roche Molecular Biochemicals). Membranes were next washed twice in washing buffer $(0 \cdot 3 \%$ Tween 20 in $0 \cdot 1 \mathrm{M}$ maleic acid, $0 \cdot 15 \mathrm{M} \mathrm{NaCl}$, $\mathrm{pH} 7 \cdot 5$ ) for $15 \mathrm{~min}$ at room temperature. Labelled DNA fragments were visualized by incubating the membranes in $225 \mu \mathrm{M}$ coumaric acid (Sigma), $1.25 \mathrm{mM}$ 3-aminophthalhydrazide (Sigma) and $0.009 \%$ hydrogen peroxide (Fisher Scientific) in $100 \mathrm{mM}$ Tris, $\mathrm{pH} 8.5$, for $1 \mathrm{~min}$ and then exposing the membranes to autoradiography film. Each membrane was sequentially exposed to multiple probes such that at least one probe hybridized to the DNA in each lane. In this manner, it was verified that sufficient DNA was present in each lane for gene detection.

Gene detection by PCR. PCR assays for detection of the exoU, exoS, exoT, exoY and $p \circ p B$ genes were performed using intact $P$. aeruginosa bacteria as a source of template chromosomal DNA. P. aeruginosa isolates were grown as individual colonies on LB agar plates. Toothpicks were used to directly inoculate a single colony into a tube containing pre-mixed PCR amplification reagents. Amplification was performed in the presence of the primers listed in Table 2. Samples were immediately placed into a thermocycler, and PCR amplification was performed using the following protocol: $94^{\circ} \mathrm{C}$ for $3 \mathrm{~min}$, then 40 cycles of $94^{\circ} \mathrm{C}$ for $30 \mathrm{~s}, 55^{\circ} \mathrm{C}$ for $30 \mathrm{~s}, 72^{\circ} \mathrm{C}$ for $2 \mathrm{~min}$, followed by $72{ }^{\circ} \mathrm{C}$ for $5 \mathrm{~min}$. PCR products were electrophoresed through agarose gels $(0.8 \%, \mathrm{w} / \mathrm{v})$ containing ethidium bromide $\left(0.5 \mu \mathrm{g} \mathrm{ml}^{-1}\right)$ and visualized using UV radiation.

DNA fingerprint analysis. The clonality of $P$. aeruginosa isolates was determined using the enterobacterial-repetitive intergenic consensus (ERIC)-PCR method (Liu et al., 1996; Louws et al., 1994; Versalovic et al., 1991). ERIC2 primers (5'AAGTAAGTGACTGGGGTGAGCG-3') were synthesized and used in PCR amplifications. Intact bacteria were used as a source of template chromosomal DNA. The following protocol was used: $94^{\circ} \mathrm{C}$ for $3 \mathrm{~min}$, then 45 cycles of $1 \mathrm{~min}$ at $94^{\circ} \mathrm{C}, 1 \mathrm{~min}$ at $45^{\circ} \mathrm{C}$ and $1 \mathrm{~min}$ at $74^{\circ} \mathrm{C}$. After the last cycle, samples were maintained at $74^{\circ} \mathrm{C}$ for $10 \mathrm{~min}$. The resulting amplified DNA fragments were electrophoresed through agarose gels $(0.8 \%, \mathrm{w} / \mathrm{v})$ containing ethidium bromide $\left(0 \cdot 5 \mu \mathrm{g} \mathrm{ml}^{-1}\right)$ and visualized using UV radiation. All fingerprint patterns contained a minimum of eight bands. A modification of the method of Liu et al. (1996) was used to interpret DNA fingerprints whereby fingerprints were considered distinct if they differed by at least three bands.

A second method of random amplified polymorphic DNA (RAPD) PCR fingerprinting, described by Mahenthiralingam et al. (1996) and Campbell et al. (2000), was used to confirm these results. This method utilizes sequence $208 \quad\left(5^{\prime}-\right.$ ACGGCCGACC-3') as a primer. Samples were amplified using the same protocol as described for ERIC-PCR fingerprinting except that the initial $94{ }^{\circ} \mathrm{C}$ for 3 min step was omitted. Fingerprints were considered distinct if they differed by at least three bands.

Statistical methods. The prevalence of effector genes amongst the different sets of clinical isolates was compared using the chi-squared test or the Fisher's exact test when appropriate.

\section{RESULTS AND DISCUSSION}

\section{Strain collection}

To investigate the distribution and prevalence of $P$. aeruginosa type III secretion genes, a large panel of clinical isolates was collected and grouped by the body site from which they were cultured. Twenty isolates (labelled UR) were obtained from the urine of patients, 20 isolates from endotracheal tube aspirates of mechanically ventilated non-CF patients (ET), 20 isolates from the sputum of patients with cystic fibrosis (CF), 20 isolates from blood (BL) and 15 isolates from wounds (WD). For comparison, 20 isolates cultured from non-hospital environmental sites (EV) were included in the analysis. In all, 115 isolates were examined.

$P$. aeruginosa frequently causes hospital epidemics that result in dissemination of a single clone to multiple patients and sites (Holder, 1977; Kropec et al., 1993). Such outbreaks may bias studies such as ours in that the epidemic clone may be overrepresented in the study sample. To eliminate this possibility, PCR-based DNA fingerprinting using ERIC2 primers was performed on all isolates (Fig. 1). Twenty $(100 \%)$ of the 20 urine isolates, $18(90 \%)$ of the 20 endotracheal isolates, 17 $(85 \%)$ of the $20 \mathrm{CF}$ respiratory isolates, $18(90 \%)$ of the 20 blood isolates, $15(100 \%)$ of the 15 wound isolates and $16(80 \%)$ of the 20 environmental isolates were nonclonal (data not shown). These findings were confirmed using a RAPD fingerprinting primer, sequence 208 (see Methods), which yielded results similar to those obtained using the ERIC2 primer (data not shown). Therefore, the great majority of these isolates represent independent strains that can be used to accurately determine the prevalence of type III secretion genes in $P$. aeruginosa. The absence of clonality among the majority of isolates indicates that most of the $P$. aeruginosa disease during the period of collection was endemic and not epidemic, and that endemic infections were due to non-clonal strains of this organism. These findings are in agreement with those of two recent studies that have concluded that isolates from the majority of endemic $P$. aeruginosa nosocomial infections are non-clonal (Bonten et al., 1999; Ruimy et al., 2001).

\section{The popB gene}

We wished to determine the percentage of clinical and environmental isolates of $P$. aeruginosa that harboured the large cluster of type III secretion genes which encodes the conserved secretion machinery. We used the $p \circ p B$ gene as a marker for the presence of the large $p s c-p c r$ exs-pop gene cluster (Frank, 1997). The $p \circ p B$ gene is located near the middle of this cluster and encodes a 


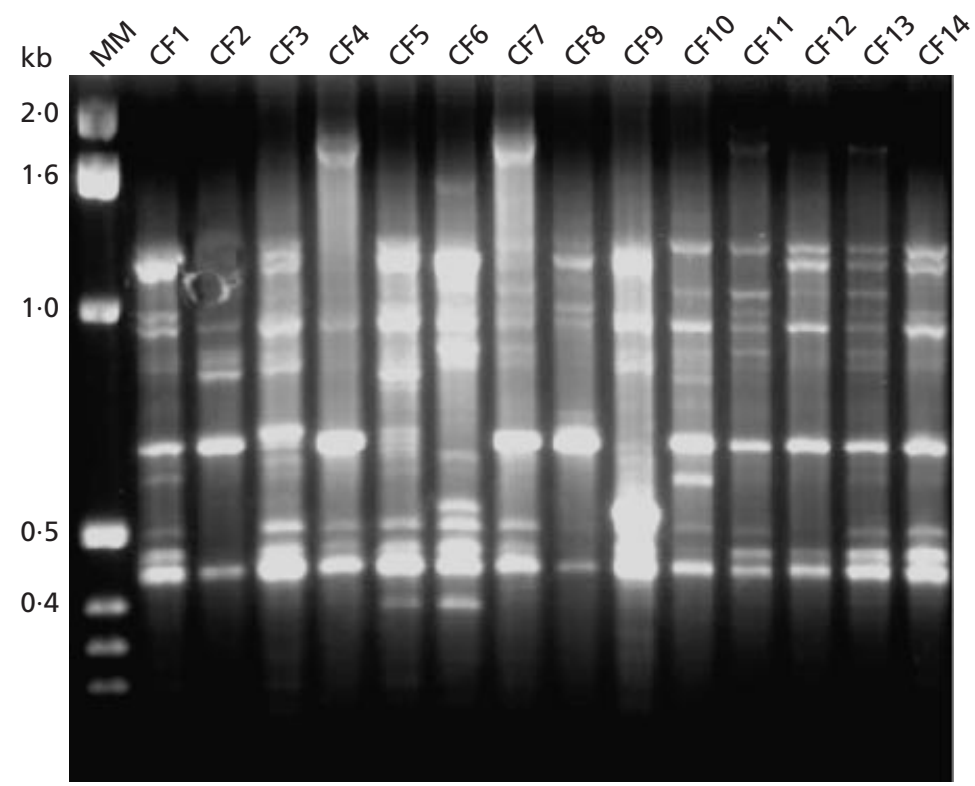

Fig. 1. Representative ERIC-PCR fingerprinting results of 14 consecutive respiratory isolates from patients with CF. Molecular mass standards are listed to the left of the panel. Differences of three or more bands between any two isolates indicated that these two isolates were non-clonal.

protein essential for proper translocation of effector proteins (Frithz-Lindsten et al., 1998). An internal probe that hybridized to the $p \circ p B$ gene was used to detect $p o p B$-like sequences in the chromosomal DNA of $P$. aeruginosa isolates (Fig. 2). All examined clinical isolates contained a single BamHI-BamHI DNA fragment that hybridized to the $p \circ p B$ probe (Table 3). Likewise, all 20 of the examined environmental isolates harboured a single Bam HI-BamHI DNA fragment that hybridized to this probe (Table 3 ). In total, $100 \%$ of 115 examined isolates contained $p o p B$-like sequences. These results were confirmed using a PCR-based detection assay that utilized primers specific for $p o p B$ (data not shown). The presence of $p \circ p B$ sequences in each of the tested isolates suggests that at least part of the $p s c-p c r$-exs-popB locus is present in all or nearly all $P$. aeruginosa isolates. These conclusions are supported by several previous reports. Alonso et al. (1999) showed that each of seven examined environmental isolates contained the $p s c J$ gene, which is also located in the large type III secretion gene cluster. Dacheux et al. (2000) noted that the exsA gene, another member of this gene cluster, was present in $28(97 \%)$ of 29 examined CF isolates.

\section{The exoT and exoS genes}

An internal probe constructed from the exoT gene was used to detect this gene in the chromosomal DNA of each isolate (Fig. 2). In addition to hybridizing to the exoT gene, this probe also occasionally yielded faint bands corresponding to the exoS gene due to the high degree of identity ( $80 \%$; Yahr et al., 1996a) between these two genes. Corresponding experiments using an exoS probe demonstrated that this probe hybridized strongly to the exoS gene and also occasionally yielded faint bands corresponding to the exoT gene. In these situations, the faint bands due to cross-hybridization were not scored. The PCR-based gene detection assay was used to confirm the presence or absence of exoTand exoS-hybridizing sequences in all isolates. One of the two PCR primers was designed to recognize DNA sequences internal to exoT while the second primer was designed to recognize unique DNA sequences flanking the exoT gene, thus ensuring specific amplification of this gene. A similar strategy was employed to uniquely amplify the exoS gene. In each case, the results of PCR amplification experiments agreed with the interpretation of Southern hybridization experiments (data not shown).

exoT-like sequences were found in $115(100 \%)$ of 115 examined isolates, suggesting that this gene is not a variable trait (Table 3). In each case, a single BamHI$B a m H I$ DNA fragment hybridized to the exoT probe. These results agree with previous observations made by Fleiszig et al. (1997), who noted that each of 14 examined isolates contained the exo $T$ gene. Interestingly, the presence of this gene in all examined environmental isolates suggests that there may be selection for the exoT gene in the non-hospital environments of $P$. aeruginosa.

Unlike exoT, exoS-like sequences were not found in all clinical isolates (Fig. 2). Sixty-seven (71\%) of 95 examined clinical isolates contained the exoS gene (Table 3). This finding is in agreement with previous reports, which had suggested that the exoS gene was not found in all $P$. aeruginosa clinical isolates. However, estimates of the actual prevalence of this gene or of ExoS production differed markedly. Woods et al. (1986) reported that of 132 tested burn, wound, urine, CF, acute pneumonia and blood isolates, $120(91 \%)$ secreted ExoS as measured by an immunoblot assay. Rumbaugh et al. (1999), who examined a collection of 25 urinary, wound and tracheal isolates, found that $24(96 \%)$ harboured the exoS gene. Fleiszig and co-workers 

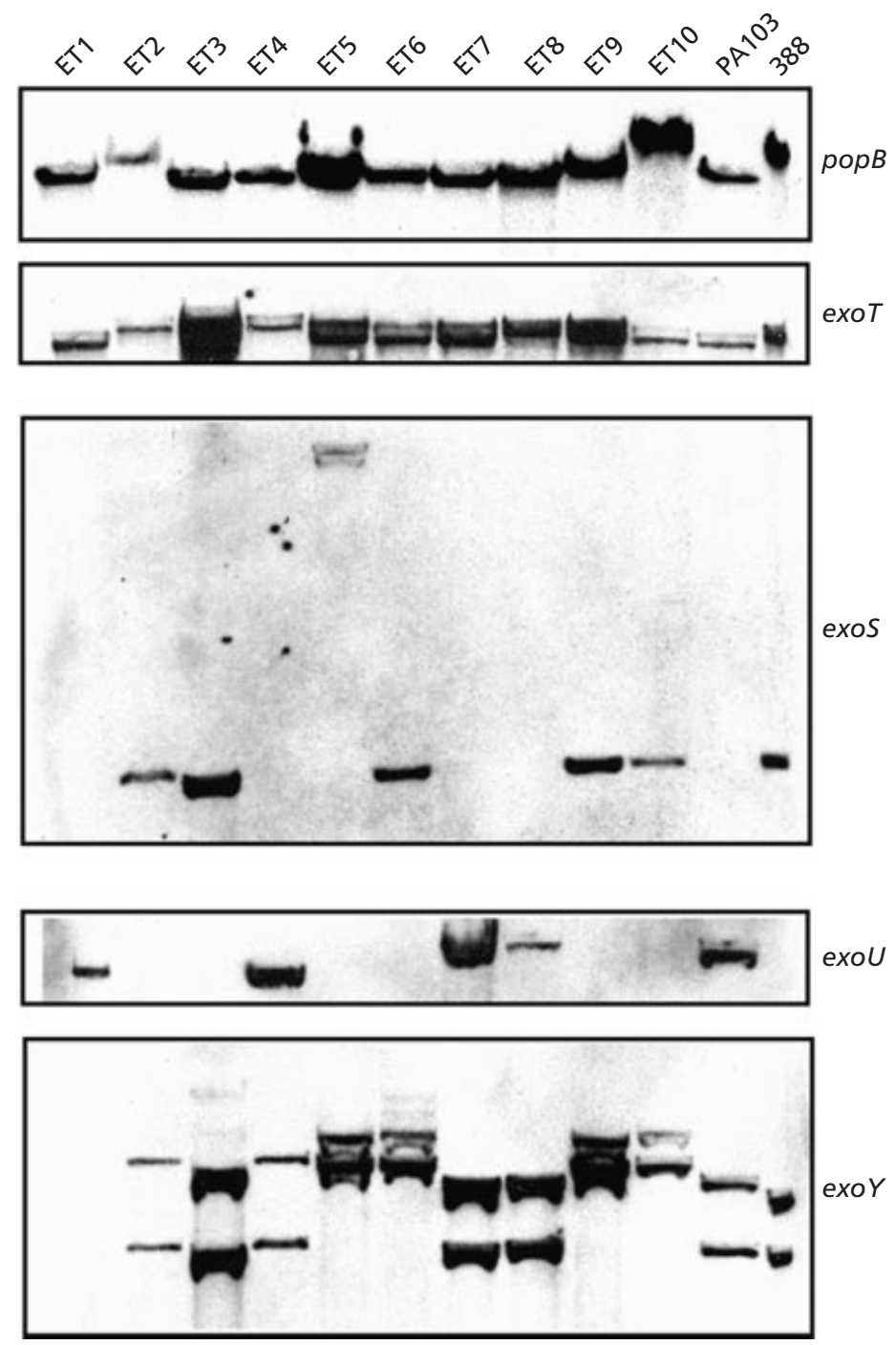

Fig. 2. Representative Southern hybridization analyses of 10 consecutive endotracheal isolates using probes for the popB, exoT, exoS, exoU and exoY genes. Chromosomal DNA was digested with $B a m H I$, electrophoresed, transferred to membrane, and exposed to labelled probes for each of the indicated genes. BamHI cuts once within the exoY gene, so two distinct DNA fragments hybridize with this probe in isolates that harbour a single copy of the exoY gene. PA103 and 388 are laboratory control strains. PA103 contains the exoU, exoT and exoY genes; 388 contains the exoS, exoT and exoY genes.

Table 3. Prevalence of type III secretion genes in $P$. aeruginosa isolates

Values are the number of isolates with the percentage of the total in parentheses.

\begin{tabular}{|c|c|c|c|c|c|c|}
\hline Source & $\begin{array}{c}\text { No. of isolates } \\
\text { examined }\end{array}$ & $\operatorname{exoU}$ & $\operatorname{exos}$ & $\operatorname{exoT}$ & $\operatorname{exo} Y$ & popB \\
\hline Urine & 20 & $7(35)$ & $14(70)$ & $20(100)$ & $14(70)$ & $20(100)$ \\
\hline Endotracheal & 20 & $5(25)$ & $15(75)$ & $20(100)$ & $19(95)$ & $20(100)$ \\
\hline Sputum (cystic fibrosis) & 20 & $2(10)$ & $17(85)$ & $20(100)$ & $18(90)$ & $20(100)$ \\
\hline Blood & 20 & $8(40)$ & $12(60)$ & $20(100)$ & $18(90)$ & $20(100)$ \\
\hline Wound & 15 & $6(40)$ & $9(60)$ & $15(100)$ & $14(93)$ & $15(100)$ \\
\hline Environment (non-hospital) & 20 & $4(20)$ & $16(80)$ & $20(100)$ & $19(95)$ & $20(100)$ \\
\hline Total & 115 & $32(28)$ & $83(72)$ & $115(100)$ & $102(89)$ & $115(100)$ \\
\hline
\end{tabular}

(Finck-Barbancon et al., 1997; Fleiszig et al., 1996) noted that only 2 of $10(20 \%)$ examined corneal isolates contained the exoS gene, although it is not clear if these isolates were randomly chosen or selected for certain phenotypic traits. The conflicting results of these studies may be due to differences in the sources of the isolates or 
to the inability of the assays to differentiate the ExoS gene or protein from the ExoT gene or protein. In any case, our findings confirm that not all isolates have the ability to produce ExoS and suggest that the actual overall prevalence of the exoS gene in clinical isolates is approximately $70 \%$.

The fraction of isolates harbouring exoS-like sequences differed somewhat between the various disease categories (Table 3). For example, whereas only 12 $(60 \%)$ of 20 examined blood isolates contained exoSlike sequences, $17(85 \%)$ of 20 CF respiratory isolates contained these sequences $(P=0 \cdot 16)$. Dacheux et al. (2000) also observed a high prevalence of the exoS gene in CF respiratory isolates. They found that $28(97 \%)$ of 29 examined CF isolates contained the exoS gene. Clinical isolates from other sources had intermediate exoS prevalence values (Table 3). For example, 15 $(75 \%)$ of 20 non-CF endotracheal isolates contained these sequences. None of these differences were statistically significant. Environmental isolates were similar to clinical isolates with respect to the prevalence of the exoS gene. Sixteen $(80 \%)$ of 20 environmental isolates contained exoS-like sequences, suggesting that ExoS is not essential for survival of $P$. aeruginosa in nature. Again, no isolates contained more than a single Bam HI$B a m H I$ DNA fragment that hybridized to the exoS probe. When both clinical and environmental isolates were combined, a total of $83(72 \%)$ of 115 examined isolates contained exoS-like sequences.

\section{The exoU gene}

exoU-hybridizing sequences were found in a minority of isolates (Table 3; Fig. 2). In total, 32 (28\%) of 115 examined clinical and environmental isolates harboured exoU-like sequences by Southern hybridization analysis. In each case, these results were confirmed using the PCR-based gene detection assay (data not shown). The prevalence of exoU-like sequences varied from $40 \%$ in wound and blood isolates, $35 \%$ in urine isolates, $25 \%$ in endotracheal isolates and $10 \%$ in CF respiratory isolates (Table 3). The difference in exoU prevalence between the blood or wound isolates and the CF isolates showed a trend towards significance $(P=0 \cdot 06)$. In comparison, $20 \%$ of environmental isolates harboured this gene. No isolate contained more than a single BamHI-BamHI DNA fragment that hybridized to the exoU probe. Dacheux et al. (2000) reported a similar value for the prevalence of the exoU gene in CF isolates. They detected the exoU gene in $3(10 \%)$ of 29 examined CF isolates. Other investigators have reported significantly different values for the prevalence of the exoU gene among bacteraemia isolates. For example, Hirakata et al. (2000) detected the exoU gene in $4(13 \%)$ of 32 bacteraemia isolates whereas Allewelt et al. (2000) detected the exoU gene in $9(64 \%)$ of 14 bacteraemia isolates. It may be that these isolates were not randomly selected or that bacteraemia isolates from patients with different underlying clinical conditions (e.g. neutropenic cancer patients vs burn patients) harbour $P$. aeruginosa isolates with different population genotypes. Fleiszig and colleagues detected the exoU gene in $8(80 \%)$ of 10 corneal isolates (Finck-Barbancon et al., 1997; Fleiszig et al., 1997), although it is unclear whether these isolates were randomly selected or chosen because of phenotypic characteristics. Corneal isolates were not examined in this study. In summary, our findings indicate that the exoU gene is present in approximately $28 \%$ of $P$. aeruginosa isolates, with the possible exception of CF isolates, which may harbour this gene less frequently.

Interestingly, exoU and exoS genes were not randomly distributed among isolates. Rather, nearly every isolate that harboured exoS-like sequences did not contain exoU-like sequences and vice versa. In fact, of the $115 P$. aeruginosa clinical and environmental isolates examined in this study, 82 contained the exoS but not the exoU gene, 31 contained the exoU but not the exoS gene, a single isolate (UR16) contained both genes, and a single isolate (CF16) contained neither of these genes (data not shown). Similar findings were reported by Fleiszig and colleagues, who noted that each of $14 P$. aeruginosa isolates contained either the exoS gene or the exoU gene (Finck-Barbancon et al., 1997; Fleiszig et al., 1997). Our results indicate that this is a near universal property of $P$. aeruginosa isolates. The reason for this unusual relationship is unclear. One possibility is that ExoU and ExoS fulfil important but redundant functions. However, ExoS and ExoU share no sequence similarity beyond the first 6 amino acids. Furthermore, whereas ExoS disrupts cell cytoskeletal elements and inhibits DNA replication (Frithz-Lindsten et al., 1997; Goehring et al., 1999; Henriksson et al., 2000; Olson et al., 1997, 1999; Pederson et al., 1999; Vallis et al., 1999; Vincent et al., 1999), ExoU is a potent cytotoxin that rapidly induces death in many cell types (Finck-Barbancon et al., 1997; Hauser et al., 1998). Therefore, any functional redundancy between these proteins is not obvious. Of note, exoU, like many of the genes that encode type III secretion effector proteins, may have been acquired by $P$. aeruginosa through horizontal transfer from another bacterial species. The $\mathrm{G}+\mathrm{C}$ content of the exoU genes is $59 \mathrm{~mol} \%$ (Hauser et al., 1998) compared to $67.2 \mathrm{~mol} \%$ for the $P$. aeruginosa chromosome as a whole (Palleroni, 1984). Also consistent with this hypothesis is that the exoU gene is located immediately adjacent to an insertion-sequence-like element (Finck-Barbancon $e t$ al., 1997; Hauser et al., 1998). Therefore, an alternative explanation for the inverse correlation between the presence of the exoU and exoS genes is that they occupy the same chromosomal locus and that the presence of exoS precludes the acquisition of exoU and vice versa. Two lines of evidence make this hypothesis unlikely. First, analysis of the genome sequence of strain PAO1 (Stover et al., 2000; the Pseudomonas Genome Project, http://www.pseudomonas.com) suggests that the exoU and exoS genes occupy distinct loci. Although the chromosome of strain PAO1 contains the exoS gene but not the exoU gene, this strain does harbour sequences identical to those flanking the exoU gene, allowing the placement of the exoU gene at approximately $1 \mathrm{Mb}$ in the chromosome. The exoS gene is located at $4.3 \mathrm{Mb}$. 
Secondly, one of the isolates examined in this study, UR16, does contain both the exoS and exoU genes, indicating that a single strain may harbour both genes. An alternative explanation is that each of these proteins may be important for $P$. aeruginosa survival in a specific but different environmental niche. The genotype of an infecting isolate may simply be indicative of the particular environmental reservoir of that isolate. Interestingly, the only isolate in this study that contained neither the exoS nor the exoU gene (CF16) was cultured from the sputum of a 23-year-old CF patient. Once colonized, CF patients often remain colonized with the same strain for many years (Romling et al., 1994). Therefore it is conceivable that either exoS or exoU was deleted from this isolate after many years of growth within the CF lung. Finally, it is possible that the exoS and exoU gene products in some way antagonize each other, resulting in a selective pressure upon $P$. aeruginosa strains to delete one or the other. Such a situation would be similar to the 'black holes' described by Maurelli et al. (1998) in Shigella spp. and enteroinvasive Escherichia coli.

\section{The exoY gene}

Southern hybridization assays indicated that $102(89 \%)$ of the 115 examined isolates contained exo $Y$-hybridizing sequences, including 19 (95\%) of 20 environmental isolates (Table 3; Fig. 2). The PCR-based gene detection assay yielded identical findings (data not shown). These results indicate that the exoY gene is present in the vast majority of $P$. aeruginosa clinical and environmental isolates and agree with those of Dacheux et al. (2000), who detected the exoY gene in $28(97 \%)$ of $29 \mathrm{CF}$ respiratory isolates. Interestingly, there was a trend towards a significant difference between the exoY gene prevalence in the urine isolates $(70 \%)$ and the endotracheal or environmental isolates $(95 \%)(P=0.09$; Table 3). The implications of this difference are unclear.

\section{exoS and exoU gene prevalence in CF and non-CF respiratory isolates}

Our results showed a trend towards a lower prevalence of the exoU gene and a higher prevalence of the exoS gene in $\mathrm{CF}$ respiratory isolates relative to non-CF isolates. That CF respiratory isolates should differ from non-CF respiratory isolates was particularly unexpected, since both sets of isolates were cultured from the same anatomical location. To further investigate this phenomenon, we analysed 20 additional respiratory isolates from patients with $\mathrm{CF}$ and 20 additional respiratory isolates from patients without CF. Whereas our initial set of 95 clinical isolates was collected in Chicago, Illinois, the additional isolates were obtained from patients in San Francisco, California, to ensure that exoS and exoU gene prevalence data did not vary with geographical location. PCR-DNA fingerprint analysis was performed on all 40 of these respiratory isolates and indicated that $80 \%$ of the CF isolates were non-clonal and that $100 \%$ of the non-CF respiratory isolates were non-clonal (data not shown). Thus the majority of these isolates are non-clonal and represent a suitable sample for determining the prevalence of the exoS and exoU genes.

Southern hybridization analysis indicated that $14(70 \%)$ of 20 non-CF isolates and $19(95 \%)$ of 20 CF isolates contained the exoS gene whereas $5(25 \%)$ of the 20 non$\mathrm{CF}$ isolates and $1(5 \%)$ of $20 \mathrm{CF}$ isolates harboured the exoU gene. These results, which were confirmed using the PCR-based gene detection assay (data not shown), are similar to those obtained using isolates from Chicago. Together, $29(73 \%)$ of 40 non-CF respiratory isolates contained the exoS gene compared to $36(90 \%)$ of $40 \mathrm{CF}$ respiratory isolates. This difference was statistically significant $(P<0 \cdot 05)$. [The difference between exoS prevalence in $\mathrm{CF}$ isolates and blood or wound isolates was also significant $(P<0 \cdot 05)$.] Southern hybridization and PCR-based assays were also used to determine the prevalence of the exoU gene in these isolates. Ten $(25 \%)$ of 40 non-CF respiratory isolates contained the exoU gene compared to $3(8 \%)$ of $40 \mathrm{CF}$ respiratory isolates, a statistically significant difference $(P<0.05)$. Thus as a population, $P$. aeruginosa strains that infect CF patients differ in their type III secretion genotypes from those that infect the respiratory tract of non-CF patients. The difference in exoU prevalence between $\mathrm{CF}$ isolates and isolates from each of the other tested disease categories was also statistically significant $(P<0 \cdot 05)$. The decreased prevalence of the exoU gene in populations of $\mathrm{CF}$ isolates is unlikely to result from deletion of the exoU gene over time because most of these isolates also harbour the exoS gene. The presence of the exoS gene suggests that these isolates had not previously harboured the exoU gene, since both these genes are seldom found in the same isolate. It is possible that there is selection against ExoU-producing strains in the CF lung. Alternatively, production of ExoS may provide $P$. aeruginosa isolates with an advantage in colonizing or persisting in the CF lung. Finally, exoS may be genetically linked to a second factor that is important in the pathogenesis of $P$. aeruginosa pulmonary colonization and infection in CF patients.

\section{Conclusions}

Taken together, our findings suggest that all $P$. aeruginosa isolates harbour at least some of the genes encoding the type III secretion apparatus. In contrast, three of the four genes encoding known $P$. aeruginosa type III effector proteins are variable traits. The exoT gene was detected in all examined isolates, but the exoY gene was present in only $89 \%$, the exoS gene in $72 \%$, and the exoU gene in $28 \%$. Interestingly, the vast majority of isolates contained either the exoS or the exoU genes but not both. In general, clinical isolates did not differ from environmental isolates in the distribution of type III effector genes. The prevalence of genes encoding effector proteins was independent of the site of infection, with the exception of CF respiratory isolates and perhaps urine isolates. CF respiratory isolates 
harboured the exoU gene less frequently and the exoS gene more frequently than did isolates from patients without CF. Urine isolates showed a trend towards a decreased prevalence of the exoY gene.

$P$. aeruginosa isolates from two cities were examined in this study and yielded very similar findings, suggesting that our results are not unique to $P$. aeruginosa populations from specific geographical locations. In addition, we have examined a collection of $35 P$. aeruginosa isolates from patients in Spain and found similar prevalences: the $p \circ p B$ and exoT genes were present in $100 \%$ of isolates, the exo Y gene in $89 \%$, the exoS gene in $66 \%$ and the exoU gene in $34 \%$ (A. R. Hauser, E. Cobb, M. Bodí, D. Mariscal, J. Vallés, J. N. Engel \& J. Rello, unpublished results). Additional studies are in progress to determine if these values are representative of $P$. aeruginosa isolates from other geographical locations, anatomical sites and host conditions.

It should be noted that this study examined only genotype, and that isolates harbouring these genes may not be competent for secretion of functional effector proteins. However, assays for in vivo secretion are cumbersome, and it remains unclear if in vitro secretion accurately predicts the ability to secrete in vivo. Therefore, these results represent an upper limit for the percentage of clinical isolates capable of secreting each type III effector protein.

We conclude that type III secretion genes are part of the virulence arsenals of nearly all $P$. aeruginosa clinical isolates. The universal distribution of these genes in clinical isolates and their importance in animal models of infection suggest that they are relevant to human disease. Future studies to determine the percentage of clinical isolates capable of secreting specific effector proteins and whether secretion correlates with disease outcomes will further elucidate the role of type III secretion in $P$. aeruginosa pathogenesis.

\section{ACKNOWLEDGEMENTS}

We thank David P. Speert, Robert V. Miller and William J. Hickey for generously supplying many of the $P$. aeruginosa environmental isolates and G. F. Brooks for facilitating collection of the Moffitt-Long Hospital isolates. We thank the technicians in the Northwestern Memorial Hospital Clinical Microbiology Laboratory for their help in collecting clinical isolates and Hank Seifert for reviewing the manuscript. We thank Barbara Kazmierczak for supplying the protocol for performing PCR directly on bacterial colonies.

This work was supported by Public Health Service grant KO8AI01524, by a Career Development Award from the Schweppe Foundation, and by an Intramural Research Grant from Northwestern Memorial Hospital.

\section{REFERENCES}

Allewelt, M., Coleman, F. T., Grout, M., Priebe, G. P. \& Pier, G. B. (2000). Acquisition of expression of the Pseudomonas aeruginosa ExoU cytotoxin leads to increased bacterial virulence in a murine model of acute pneumonia and systemic spread. Infect Immun 68, 3998-4004.
Alonso, A., Rojo, F. \& Martinez, J. L. (1999). Environmental and clinical isolates of Pseudomonas aeruginosa show pathogenic and biodegradative properties irrespective of their origin. Environ Microbiol 1, 421-430.

Bonten, M. J. M., Bergmans, C. J. J., Speijer, H. \& Stobberingh, E. E. (1999). Characteristics of polyclonal endemicity of Pseudomonas aeruginosa colonization in intensive care units. Am J Respir Crit Care Med 160, 1212-1219.

Campbell, M., Mahenthiralingam, E. \& Speert, D. P. (2000). Evaluation of random amplified polymorphic DNA typing of Pseudomonas aeruginosa. J Clin Microbiol 38, 4614-4615.

Chen, W.-P. \& Kuo, T.-T. (1993). A simple and rapid method for the preparation of gram-negative bacterial genomic DNA. Nucleic Acids Res 21, 2260.

Coburn, J. \& Frank, D. (1999). Macrophages and epithelial cells respond differently to the Pseudomonas aeruginosa type III secretion system. Infect Immun 67, 3151-3154.

Cowell, B. A., Chen, D. Y., Frank, D. W., Vallis, A. J. \& Fleiszig, S. M. J. (2000). ExoT of cytotoxic Pseudomonas aeruginosa prevents uptake by corneal epithelial cells. Infect Immun 68, 403-406.

Dacheux, D., Toussaint, B., Richard, M., Brochier, G., Croize, J. \& Attree, I. (2000). Pseudomonas aeruginosa cystic fibrosis isolates induce rapid, type III secretion-dependent, but ExoU-independent, oncosis of macrophages and polymorphonuclear neutrophils. Infect Immun 68, 2916-2924.

Fagon, J. Y., Chastre, J., Domart, Y., Trouillet, J. L., Pierre, J., Carne, C. \& Gibert, C. (1989). Nosocomial pneumonia in patients receiving continuous mechanical ventilation. Am Rev Respir Dis 139, 877-884.

Fagon, J.-Y., Chastre, J., Hance, A. J., Montravers, P., Novara, A. \& Gibert, C. (1993). Nosocomial pneumonia in ventilated patients: a cohort study evaluating attributable mortality and hospital stay. Am J Med 94, 281-288.

Finck-Barbancon, V., Goranson, J., Zhu, L., Sawa, T., WienerKronish, J. P., Fleiszig, S. M. J., Wu, C., Mende-Mueller, L. \& Frank, D. (1997). ExoU expression by Pseudomonas aeruginosa correlates with acute cytotoxicity and epithelial injury. Mol Microbiol 25, 547-557.

Fitzsimmons, S. C. (1993). The changing epidemiology of cystic fibrosis. J Pediatr 122, 1-9.

Fleiszig, S. M. J., Zaidi, T. S., Preston, M. J., Grout, M., Evans, D. J. \& Pier, G. B. (1996). Relationship between cytotoxicity and corneal epithelial cell invasion by clinical isolates of Pseudomonas aeruginosa. Infect Immun 64, 2288-2294.

Fleiszig, S. M. J., Wiener-Kronish, J. P., Miyazaki, H., Vallas, V., Mostov, K., Kanada, D., Sawa, T., Yen, T. S. B. \& Frank, D. (1997). Pseudomonas aeruginosa-mediated cytotoxicity and invasion correlate with distinct genotypes at the loci encoding exoenzyme S. Infect Immun 65, 579-586.

Fluit, A. C., Verhoef, J. \& Schmitz, F. J. (2000). Antimicrobial resistance in European isolates of Pseudomonas aeruginosa. European SENTRY participants. Eur J Clin Microbiol Infect Dis 19, 370-374.

Frank, D. (1997). The exoenzyme S regulon of Pseudomonas aeruginosa. Mol Microbiol 26, 621-629.

Frithz-Lindsten, E., Du, Y., Rosqvist, R. \& Forsberg, A. (1997). Intracellular targeting of exoenzyme $S$ of Pseudomonas aeruginosa via type III-dependent translocation induces phagocytosis resistance, cytotoxicity and disruption of actin microfilaments. Mol Microbiol 25, 1125-1139. 
Frithz-Lindsten, E., Holmstrom, A., Jacobsson, L., Soltani, M., Olsson, J., Rosqvist, R. \& Forsberg, A. (1998). Functional conservation of the effector protein translocators PopB/YopB and PopD/YopD of Pseudomonas aeruginosa and Yersinia pseudotuberculosis. Mol Microbiol 29, 1155-1165.

Garrity-Ryan, L., Kazmierczak, B., Kowal, R., Comolli, J., Hauser, A. \& Engel, J. N. (2000). The arginine finger domain of ExoT contributes to actin cytoskeleton disruption and inhibition of internalization of Pseudomonas aeruginosa by epithelial cells and macrophages. Infect Immun 68, 7100-7113.

Gilardi, G. L. (1991). Pseudomonas and related genera. In Manual of Clinical Microbiology, pp. 429-441. Edited by A. Balows, W. J. Hausler, Jr, K. L. Herrmann, H. D. Isenberg \& H. J. Shadomy. Washington, DC: American Society for Microbiology.

Goehring, U.-M., Schmidt, G., Pederson, K. J., Aktories, K. \& Barbieri, J. T. (1999). The N-terminal domain of Pseudomonas aeruginosa exoenzyme $\mathrm{S}$ is a GTPase-activating protein for Rho GTPases. J Biol Chem 274, 36369-36372.

Hanberger, H., Garcia-Rodriguez, J.-A., Gobernado, M., Goossens, H., Nilsson, L. E. \& Struelens, M. J. (1999). Antibiotic susceptibility among aerobic gram-negative bacilli in intensive care units in 5 European countries. JAMA (J Am Med Assoc) 281, $67-71$.

Hauser, A. R. \& Engel, J. N. (1999). Pseudomonas aeruginosa induces type III secretion-mediated apoptosis of macrophages and epithelial cells. Infect Immun 67, 5530-5537.

Hauser, A. R., Kang, P. J. \& Engel, J. (1998). PepA, a novel secreted protein of Pseudomonas aeruginosa, is necessary for cytotoxicity and virulence. Mol Microbiol 27, 807-818.

Henriksson, M. L., Rosqvist, R., Telepnev, M., Wolf-Watz, H. \& Hallberg, B. (2000). Ras effector pathway activation by epidermal growth factor is inhibited in vivo by exoenzyme S ADPribosylation of Ras. Biochem J 347, 217-222.

Hirakata, Y., Finlay, B. B., Simpson, D. A., Kohno, S., Kamihira, S. \& Speert, D. P. (2000). Penetration of clinical isolates of Pseudomonas aeruginosa through MDCK epithelial cell monolayers. $J$ Infect Dis 181, 765-769.

Holder, I. A. (1977). Epidemiology of Pseudomonas aeruginosa in a burns hospital. In Pseudomonas aeruginosa: Ecological Aspects and Patient Colonization, pp. 77-95. Edited by V. M. Young. New York: Raven Press.

Kang, P. J., Hauser, A. R., Apodaca, G., Fleiszig, S., WienerKronish, J., Mostov, K. \& Engel, J. N. (1997). Identification of Pseudomonas aeruginosa genes required for epithelial cell injury. Mol Microbiol 24, 1249-1262.

Krall, R., Schmidt, G., Aktories, K. \& Barbieri, J. T. (2000). Pseudomonas aeruginosa ExoT is a Rho GTPase-activating protein. Infect Immun 68, 6066-6068.

Kropec, A., Huebner, J., Riffel, M., Bayer, U., Benzing, A., Geiger, K. \& Daschner, F. D. (1993). Exogenous or endogenous reservoirs of nosocomial Pseudomonas aeruginosa and Staphylococcus aureus infections in a surgical intensive care unit. Intensive Care Med 19, 161-165.

Kurahashi, K., Kajikawa, O., Sawa, T., Ohara, M., Gropper, M. A., Frank, D. W., Martin, T. R. \& Wiener-Kronish, J.P. (1999). Pathogenesis of septic shock in Pseudomonas aeruginosa pneumonia. J Clin Invest 104, 743-750.

Liu, Y., Davin-Regli, A., Bosi, C., Charrel, R. N. \& Bollet, C. (1996). Epidemiological investigation of Pseudomonas aeruginosa nosocomial bacteremia isolates by PCR-based DNA fingerprinting analysis. J Med Microbiol 45, 359-365.
Louws, F. J., Fulbright, D. W., Stephens, C. T. \& de Bruijn, F. J. (1994). Specific genomic fingerprints of phytopathogenic Xanthomonas and Pseudomonas pathovars and strains generated with repetitive sequences and PCR. Appl Environ Microbiol 60, 2286-2295.

Mahenthiralingam, E., Campbell, M. E., Foster, J., Lam, J. S. \& Speert, D. P. (1996). Random amplified polymorphic DNA typing of Pseudomonas aeruginosa isolates recovered from patients with cystic fibrosis. J Clin Microbiol 34, 1129-1135.

Maurelli, A. T., Fernandez, R. E., Bloch, C. A., Rode, C. K. \& Fasano, A. (1998). "Black holes" and bacterial pathogenicity: a large genomic deletion that enhances the virulence of Shigella spp. and enteroinvasive Escherichia coli. Proc Natl Acad Sci U S A 95, 3943-3948.

National Nosocomial Infections Surveillance System (1996). National Nosocomial Infections Surveillance report, data summary from October 1986-April 1996, issued May 1996. Am J Infect Control 24, 380-388.

Olson, J. C., McGuffie, E. M. \& Frank, D. W. (1997). Effects of differential expression of the 49-kilodalton exoenzyme $S$ by Pseudomonas aeruginosa on cultured eukaryotic cells. Infect Immun 65, 248-256.

Olson, J. C., Fraylick, J. E., McGuffie, E. M., Dolan, K. M., Yahr, T. L., Frank, D. W. \& Vincent, T. S. (1999). Interruption of multiple cellular processes in HT-29 epithelial cells by Pseudomonas aeruginosa exoenzyme S. Infect Immun 67, 2847-2854.

Palleroni, N. J. (1984). Pseudomonadaceae. In Bergey's Manual of Systematic Bacteriology, pp. 141-219. Edited by N. R. Kreig \& J. G. Holt. Baltimore: Williams \& Wilkins.

Pedersen, S. S., Jensen, T., Pressler, T., Hoiby, N. \& Rosendal, K. (1986). Does centralized treatment of cystic fibrosis increase the risk of Pseudomonas aeruginosa infection? Acta Paediatr Scand 75, 840-845.

Pederson, K. J., Vallis, A. J., Aktories, K., Frank, D. W. \& Barbieri, J. T. (1999). The amino-terminal domain of Pseudomonas aeruginosa ExoS disrupts actin filaments via small-molecularweight GTP-binding proteins. Mol Microbiol 32, 393-401.

Pennington, J. E. (1995). Nosocomial respiratory infections. In Principles and Practice of Infectious Diseases, pp. 2599-2607. Edited by G. L. Mandell, J. E. Bennett \& R. Dolin. New York: Churchill Livingstone.

Romling, U., Fiedler, B., Bosshammer, J., Grothues, D., Greipel, J., von der Hardt, H. \& Tummler, B. (1994). Epidemiology of chronic Pseudomonas aeruginosa infection in cystic fibrosis. J Infect Dis 170, 1616-1621.

Ruimy, R., Genauzeau, E., Barnabe, C., Beaulieu, A., Tibayrenc, M., Andremont, A. \& The P. aeruginosa Study Group (2001). Genetic diversity of Pseudomonas aeruginosa strains isolated from ventilated patients with nosocomial pneumonia, cancer patients with bacteremia, and environmental water. Infect Immun 69, 584-588.

Rumbaugh, K. P., Hamood, A. N. \& Griswold, J. A. (1999). Analysis of Pseudomonas aeruginosa clinical isolates for possible variations within the virulence genes exotoxin A and exoenzyme S. J Surg Res 82, 95-105.

Salyers, A. A. \& Whitt, D. D. (1994). Bacterial Pathogenesis: a Molecular Approach. Washington, DC: American Society for Microbiology.

Stover, C. K., Pham, X. Q., Erwin, A. L. \& 28 other authors (2000). Complete genome sequence of Pseudomonas aeruginosa PAO1, an opportunistic pathogen. Nature 406, 959-964. 
Vallis, A. J., Finck-Barbancon, V., Yahr, T. L. \& Frank, D. W. (1999). Biological effects of Pseudomonas aeruginosa type III-secreted proteins on CHO cells. Infect Immun 67, 2040-2044.

Versalovic, J., Koeuth, T. \& Lupske, J. R. (1991). Distribution of repetitive DNA sequences in eubacteria and application to fingerprinting of bacterial genomes. Nucleic Acids Res 19, 6823-6831.

Vincent, T. S., Fraylick, J. E., McGuffie, E. M. \& Olson, J. C. (1999). ADP-ribosylation of oncogenic Ras proteins by Pseudomonas aeruginosa exoenzyme $S$ in vivo. Mol Microbiol 32, 1054-1064.

Vogel, H. J. \& Bonner, D. M. (1956). Acetylornithinase of Escherichia coli partial purification and some properties. J Biol Chem 218, 97-106.

Woods, D. E., Schaffer, M. S., Rabin, H. R., Campbell, G. D. \& Sokol, P. A. (1986). Phenotypic comparison of Pseudomonas aeruginosa strains isolated from a variety of clinical sites. J Clin Microbiol 24, 260-264.

Yahr, T. L., Barbieri, J. T. \& Frank, D. W. (1996a). Genetic relationship between the 53- and 49-kilodalton forms of exoenzyme S from Pseudomonas aeruginosa. J Bacteriol 178, 1412-1419.

Yahr, T. L., Goranson, J. \& Frank, D. W. (1996b). Exoenzyme S of Pseudomonas aeruginosa is secreted by a type III secretion pathway. Mol Microbiol 22, 991-1003.

Yahr, T. L., Vallis, A. J., Hancock, M. K., Barbieri, J. T. \& Frank, D. W. (1998). ExoY, an adenylate cyclase secreted by the Pseudomonas aeruginosa type III system. Proc Natl Acad Sci U S A 95, 13899-13904.

Received 6 March 2001; revised 15 May 2001; accepted 12 June 2001. 